\title{
Non-destructive Fluorescence Spectroscopy as a Tool for Discriminating Between Olive Oils According to Agronomic Practices and for Assessing Quality Parameters
}

\author{
Elísabet Martín-Tornero ${ }^{1}$ (D) - Antonio Fernández ${ }^{2}$ · Juan Manuel Pérez-Rodriguez ${ }^{3,4}$ - Isabel Durán-Merás ${ }^{5}$. \\ María Henar Prieto $^{3,4}$. Daniel Martín-Vertedor ${ }^{2,4}$
}

Received: 16 April 2021 / Accepted: 9 August 2021 / Published online: 4 September 2021

(c) The Author(s) 2021

\begin{abstract}
A non-destructive fluorescence method combined with chemometric algorithms has been developed for discriminating between olive oils. The excitation-emission matrices (EEMs) of two olive oil varieties (Arbosana and Oliana) from two crop seasons, which had undergone two different irrigation treatments (control irrigation strategy and regulated deficit irrigation (RDI)), were recorded. EEMs were analysed using parallel factor analysis (PARAFAC), followed by linear discriminant analysis (LDA) incorporating three PARAFAC components. This analysis was able to discriminate between olive oils according to crop season ( $100 \%$ of predictions in the validation set were correct) and variety (100\% of predictions were correct). Moreover, good discrimination ( $80 \%$ of correct predictions) was also achieved when examining olive oils belonging to the same variety but submitted to two different irrigation treatments. Further, the olive oil quality parameters obtained using conventional methods were compared with those obtained using unfolded partial least squares (U-PLS). Good correlation coefficients were obtained for Rancimat hours $(r=0.87), \mathrm{K}_{270}(r=0.75)$ and total polyphenol content $(r=0.94)$.
\end{abstract}

Keywords Olive oil · Excitation/emission matrices $\cdot$ PARAFAC $\cdot$ PARAFAC-LDA $\cdot$ U-PLS

\section{Introduction}

Olive oil is considered one of the key products present in the Mediterranean diet due to its nutritional and antioxidant profile, as well as to its important role in the economy of the producing countries. It is well known that olive oil induces

Elísabet Martín-Tornero

elisabetmt@unex.es

1 Department of Agricultural and Forestry Engineering, School of Agrarian Engineering, University of Extremadura, Avda. Adolfo Suárez, s/n, 06007 Badajoz, Spain

2 Technological Institute of Food and Agriculture CICYTEX-INTAEX, Junta of Extremadura, Avda. Adolfo Suárez, s/n, 06007 Badajoz, Spain

3 CICYTEX, Junta of Extremadura, Finca La Orden, Guadajira, 06187 Badajoz, Spain

4 Research Institute of Agricultural Resources (INURA), Avda. de la Investigación, s/n, Campus Universitario, 06006 Badajoz, Spain

5 Department of Analytical Chemistry, Faculty of Science, University of Extremadura, 06006 Badajoz, Spain important health benefits due to its specific composition of unsaturated fatty acids, polyphenols, vitamin E, carotenoids, sterols, etc. Nowadays, consumers are highly interested in adding antioxidant compounds to their daily diet due to the short and long-term benefits (Difonzo et al. 2017) they offer, for instance, in preventing and reducing certain gastrointestinal diseases such as colon cancer (Bassani et al. 2016; Terzuoli et al. 2016).

The concentration of these bioactive compounds in olive oil is deeply influenced by the cultivar type, maturation stage, agroclimatic conditions (rainfall and/or water stress) and agronomical practices (Dagdelen et al. 2013; Franco et al. 2014). Recent research has indicated that cultivar-based tailor-made irrigation strategies may upgrade final olive oil quality (Machado et al. 2013). In addition, adoption of a regulated deficit irrigation strategy is recommended in order to optimize the vigour of olive trees in highly intensive olive groves (Lavee et al. 2007). Moreover, Cabrera-Bañegil et al. (Cabrera-Bañegil et al. 2018) found that polyphenol concentration is significantly affected by the irrigation conditions. Their study indirectly revealed that a higher polyphenolic concentration was found in table olive paste produced from 
plants subjected to higher water stress. In other words, irrigation deficits promote major phenolic compound synthesis.

The high production of olive oil has led to the development of reliable and accurate methods for its authentication which consider aspects such as adulteration, mislabelling and misleading characterizations and presentations of origin (Guimet et al. 2005b). The constant development of better products leads to the need for new, faster and more reliable technologies for quality control. Moreover, due to an increased attention over pollution, there is a demand for eco-friendly analytical techniques, and, in this sense, fluorescence spectroscopy meets the requests for green analytical techniques because it is reagent free.

In addition, this technique is easy to use, rapid and multiparametric and presents a low analytical cost. Consequently, a significantly higher number of studies are emerging to validate the application of fluorescence, specifically excitation-emission matrices (EEMs), for the characterization and evaluation of parameters pertaining to olive oil. EEMs have been applied in combination with chemometric tools to discriminate between olive oils coming from two different denomination of origin regions in Spain (Guimet et al. 2005a), between three types of commercial Spanish olive oils used for human consumption (virgin, pure and olive pomace oil) (Guimet et al. 2004a) and between olive oils with low and high total polyphenol content (Squeo et al. 2019).

Furthermore, applications using total synchronous fluorescence spectroscopy and excitation-emission fluorescence spectroscopy (EEFS) have been developed to monitor changes in olive oil during storage under different conditions (Sikorska et al. 2008; Domínguez Manzano et al. 2019) and in the chemical makeup of thermoxidized virgin olive oil (Tena et al. 2012). Further, Duran-Merás et al. (Durán Merás et al. 2018) demonstrated that it was possible to detect adulterations in extra virgin olive oil as well as to quantify the level of adulteration.

This technique has been also applied to olive oil with the aim of quantification. For instance, it has been used to quantify secondary oxidation products (K270) in extra virgin, virgin, pure olive oil and olive fruit (Guimet et al. 2005b), in addition to the degree of adulteration (Guimet et al. 2005c) and total polyphenol content (Squeo et al. 2019).

In this study, two different olive varieties (Arbosana and Oliana) were selected and submitted to two irrigation regimes during two olive seasons. The aim of this work was to outline the suitability and effectiveness of fluorescence spectroscopy combined with chemometrics algorithms in discriminating between extra virgin olive oils (EVOO) according to their age, variety and the irrigation conditions submitted to. To the best of our knowledge, the discrimination of olive oils according to irrigation treatment has not been examined in any previous study. Moreover, we sought to examine the use of this methodology for quantifying oxidative stability and total polyphenol content. Different algorithms were used in support of the aforementioned methodology: parallel factor analysis (PARAFAC) for exploratory analysis, PARAFAC combined with linear discriminant analysis (PARAFAC-LDA) for classification and unfoldedpartial least squares (U-PLS) for quantification.

\section{Material and Methods}

\section{Experimental Olive Cultivar}

The study was performed with Arbosana and Oliana variety olives from an experimental olive grove (Olea europaea L.) which were harvested during the 2016/17 and 2017/18 olive seasons. The olive grove is located at the research centre " $\mathrm{La}$ Orden-CICYTEX” (Badajoz, Spain) (38 $\left.8^{\circ} 51^{\prime} \mathrm{N}, 6^{\circ} 40^{\prime} \mathrm{O}\right)$. The plot was planted in 2012 with a density of 1975 trees/ha $(1.35 \mathrm{~m} \times 3.75 \mathrm{~m})$ and is found at an altitude of $200 \mathrm{~m}$ above sea level. Rainfall during the 2016/2017 and 2017/2018 crop seasons was 489 and $275 \mathrm{~mm}$, respectively.

The experiment had a split-plot factorial design with eight replications, with the main plot consisting of two levels pertaining to the two types of irrigation and the two varieties making up two subplots. The grove consisted of three rows of trees with 6 trees per row. The four central trees were used as experimental trees, and the rest were used as guard trees.

The influence of different irrigation treatments and olive varieties on analytical parameters was studied. A total of 64 samples were collected manually from the selected central trees ( 8 blocks $\times 2$ irrigations $\times 2$ varieties $\times 2$ growing seasons) in perfect sanitary conditions. These samples were collected during November with a maturity index of 2.5 (veraison) (Uceda and Frías 1975). This maturity index guarantees the maximum fat content in Arbosana and Oliana varieties.

Random fruit samples were picked in the morning from different sides of trees with the same ripeness index. All samples for all treatments weighed $10 \mathrm{~kg}$. Samples were transported to the laboratory in ventilated storage trays. Oil was then extracted within $24 \mathrm{~h}$.

\section{Irrigation Treatments}

Two irrigation treatments were applied: (i) Control: an irrigation strategy designed to ensure optimal conditions according to the overall water requirements of the grove over the course of the irrigation season (Orgaz et al. 2006); (ii) regulated deficit irrigation (RDI): applied 52\% and 66\% of the treatment applied in Control in 2016/17 and 2017/18, respectively.

In the RDI treatment, different irrigation doses were applied depending on the growth stage of the olive fruit 
and seasonal sensitivity to water deficit. The RDI amount of water was adjusted by stem water potential $\left(\psi_{\mathrm{s}}\right)$ according to the olive phenologic stage. Thus, this parameter was adjusted at $-1.4 \mathrm{MPa}$ from sprouting to bone hardening. From veraison to harvesting, the $\psi_{\mathrm{s}}$ was set at $-1.6 \mathrm{MPa}$ (Prieto et al. 2016).

The different climatic conditions of each campaign caused variations in the final doses applied in the irrigation treatments. The total amount of water used in the Control and RDI treatments in the 2016/2017 campaign was 400 and $190 \mathrm{~mm}$, respectively. In 2017/2018, the amount of water used was 516 and $341 \mathrm{~mm}$ for Control and RDI, respectively.

\section{Oil Extraction}

An Abencor analyser (MC2 Engineering Systems, Seville, Spain) was used for oil extraction (Martínez et al. 1975). Olives were crushed with a hammer mill $24 \mathrm{~h}$ after harvesting and slowly mixed for $30 \mathrm{~min}$ at $25^{\circ} \mathrm{C}$. Following this, a paste was obtained which was centrifuged at $1438 \mathrm{~g}$ for $3 \mathrm{~min}$. The oil was then decanted and stored in ambercoloured glass bottles at $4{ }^{\circ} \mathrm{C}$.

All examined olive oils showed values below the maximum established limits for determined variables (free acidity $\leq 0.8^{\circ}$; peroxide index $\leq 20$ meq $\mathrm{O}_{2} / \mathrm{kg} ; \mathrm{K}_{270} \leq 0.22$; $\mathrm{K}_{232} \leq 2.5$ ). All included oils could therefore be labelled as "extra virgin", according to Regulation EC/1989/2003 (EEC 2003).

\section{Quality Index}

\section{Oxidative Stability}

The Rancimat test (Metrohm, Herisau, Switzerland) was used to analyse the oxidative stability of the oil (Gutiérrrez 1989). The oil was submitted to a temperature of $100{ }^{\circ} \mathrm{C}$ in order to observe changes in conductivity. Results were presented according to induction time (h). Longer induction times indicate greater oxidative stability of the oil.

\section{Total Polyphenols}

Total polyphenol content of the olive oil was evaluated according to the Folin-Ciocalteu colorimetric method using caffeic acid as a standard (Delgado-Adámez et al. 2014). Two millilitres of the extract was mixed in a vortex (VWR® Vortex Mixers, $230 \mathrm{~V}$ ) following the addition of $1 \mathrm{~mL}$ of Folin-Ciocalteu reagent (Panreac, Barcelona, Spain), $2 \mathrm{~mL}$ of saturated $\mathrm{Na}_{2} \mathrm{CO}_{3}$ and $15 \mathrm{~mL}$ of distilled water. The mix was incubated for $90 \mathrm{~min}$, and spectrophotometric absorbance was measured at $725 \mathrm{~nm}$ against a blank solution using a UV-Vis Spectrophotometer (GENESYS ${ }^{\mathrm{TM}}$ 10, from Thermo Scientific ${ }^{\mathrm{TM}}$ ). Samples were measured in triplicate, and a calibration curve was plotted against caffeic acid concentration ( $\geq 99 \%$ purity, from ExtraSynthese). Outcomes were expressed as $\mathrm{mg}$ of caffeic acid equivalent per $\mathrm{kg}$ of oil.

\section{Absorbance at 270 and $232 \mathrm{~nm}$}

The methodology used to analyse absorbance at 232 and $270 \mathrm{~nm}$ is described in Regulation (EEC) $\mathrm{n}^{\circ} 2568 / 91$ and its subsequent amendments. All olive oil samples were analysed in duplicate.

\section{Statistical Analysis}

Two-way ANOVA was performed in order to consider the effects and/or interactions of crop season and irrigation treatment within each olive variety using SPSS 22.0 software (SPSS Inc., Chicago, IL, USA). Data was analysed via ANOVA. Tukey's test was applied beforehand to verify normality and homogeneity of variance. In cases where the interaction was significant $(p<0.05)$, the results were expressed as the mean \pm SD of each crop season in the variable studied.

\section{Excitation-Emission Matrices (EEMs)}

Olive oil fluorescence measurements were performed using a Varian Model Cary Eclipse fluorescence spectrophotometer (Agilent Technologies, Madrid, Spain), and Cary Eclipse 1.2 software was used for data acquisitions. Measurements were recorded in a 10-mm quartz cell at room temperature. Excitation and emission slit widths were set at 5 and $5 \mathrm{~nm}$, respectively. Photomultiplier tube sensitivity was fixed at $750 \mathrm{~V}$.

Excitation-emission matrices (EEMs) were assembled to measure a set of emission spectra over a range of excitation wavelengths. Selected excitation wavelengths ranged from 300 to $400 \mathrm{~nm}$ and increased in $5 \mathrm{~nm}$ increments. At each excitation wavelength, emission spectra were recorded for every $2 \mathrm{~nm}$ between 410 and $625 \mathrm{~nm}$. Each sample was measured in duplicate. Data were saved in ASCII format and transferred to a PC for the chemometric analysis described in the next section.

\section{Chemometric Analysis}

Second-order data were first processed via PARAFAC (Bro 1997). This algorithm broke down the data according to excitation and emission loadings, and into their relative component concentrations or scores. In order to select the optimum number of components, core consistency diagnostic criterion (CORCONDIA) (Bro and Kiers 2003), residual analysis (Bro 1997) and physiognomy of the loading were employed. 
Given that all concentrations and spectral values are always positive, non-negative constraints were applied to all modes with the aim of obtaining a realistic solution. PARAFAC scores were employed for classification via LDA (Berrueta et al. 2007).

U-PLS (Wold et al. 1987) regression was selected to develop quantitative models between EEMs and chemical parameters. In order to select the optimum number of components, cross-validation was employed alongside with the Haaland and Thomas criterion (Haaland and Thomas 1988). The optimal number of components is indicated when a PRESS value that is not statistically different from the minimum PRESS value (F-ratio probability falling below 0.75 ) is achieved.

Samples were divided into two sets for the development of classification (PARAFAC-LDA) and quantification models (U-PLS). The calibration set (70\% of all samples) was used to build a model of known variables and conduct crossvalidation. The validation set (30\% of all samples) was used for external validation of the model. Samples were randomly divided between sets.

Data were processed using MATLAB software (MATLAB R2016b). PARAFAC and U-PLS were carried out by means of the graphical interface MVC2 (http://www.iquirconicet.gov.ar/descargas/mvc2.rar") (Olivieri et al. 2009), and the classification toolbox (https://michem.unimib.it/ download/matlab-toolboxes/) was used for LDA calculations (Ballabio and Consonni 2013).

\section{Results and Discussion}

\section{Effect of Different Irrigation Treatments on the Quality Index}

Table 1 summarizes the quality parameters for Arbosana and Oliana varieties EVOOs produced from superhigh-density olive hedgerow orchards subjected to different irrigation treatments. As can be seen, regardless of the variety and of the irrigation treatment, the olive oil production in 2017/2018 crop year (between 2445 and $3473 \mathrm{~kg} / \mathrm{ha}$ ) was higher than the one obtained in 2016/2017 (between 1453 and 2752 kg/ha). Furthermore, Arbosana was the variety with more olive oil production in both campaigns. With respect to the influence of the irrigation treatment in the olive oil yield, a significant difference was observed between the two treatments, being the Control treatment the one which present higher levels of productions.

Also, other parameters such as total polyphenol content, oxidative stability, and K270 and K232 values have been included. The analysis revealed a significant

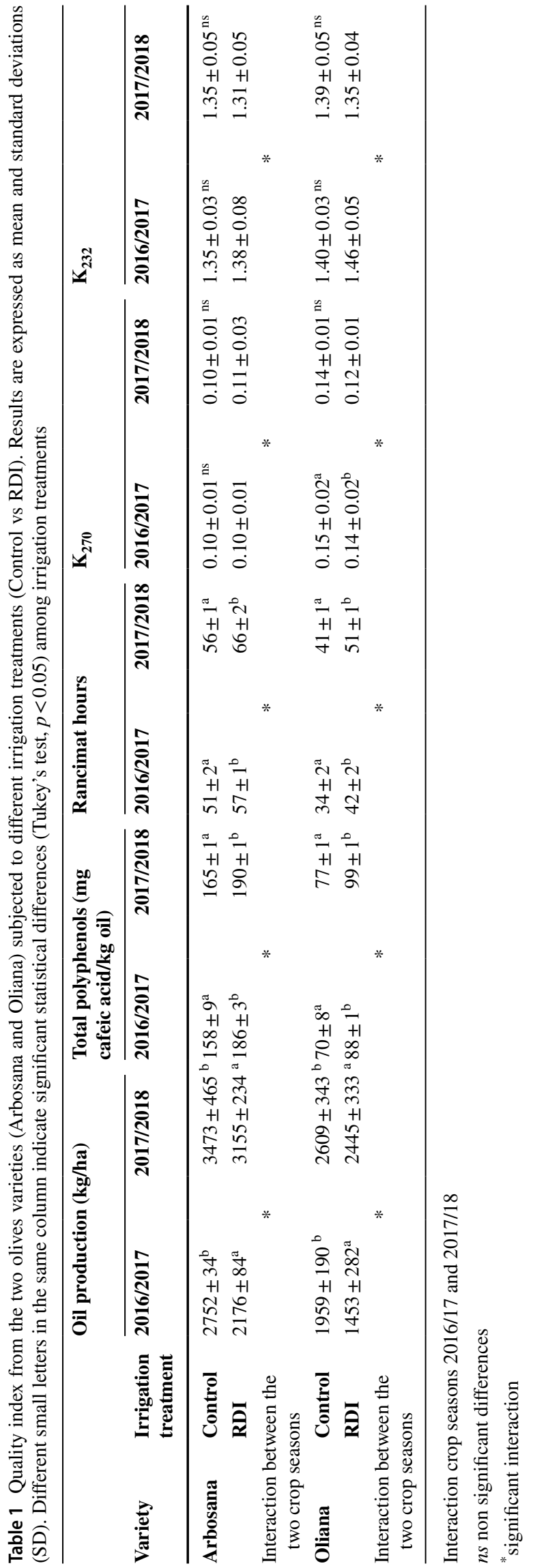


interaction between crop season and irrigation treatment in both varieties. Therefore, data from the two crop seasons are presented separately.

In this sense, higher values of total polyphenols and oxidative stability were observed in oil produced from the $2017 / 2018$ crop season. This may be explained by the lower rainfall seen during this year, which only amounted to $44 \%$ of the rain seen during the previous season. Differences in absorbance at 270 and $232 \mathrm{~nm}$ were not seen between the two seasons, and these values were within established limits $\left(\mathrm{K}_{270} \leq 0.22 ; \mathrm{K}_{232} \leq 2.5\right)$. Consequently, parameters for all of the studied oils were considered within the category of "extra virgin olive oil" as stipulated by Regulation (EEC) 2568/91.

Irrigation regimen had a strong influence on total polyphenol concentration and oxidative stability; however, it had no effect on either of the K parameters. Total polyphenol content decreased as irrigation dose increased. Statistical differences were observed between irrigation treatments, crop seasons and cultivar varieties. The highest values were exhibited by EVOOs produced from Arbosana variety trees with a below RDI dose of irrigation, $190.3 \mathrm{mg}$ Caf./kg oil, and subjected to an induction time of $66 \mathrm{~h}$. On the other hand, the lowest values were shown in EVOO from trees watered with a greater quantity of water. The greatest differences were observed in relation to the Oliana variety when both parameters were more than $18 \%$ below RDI relative to control conditions.

This trend is consistent with findings reported by other authors. In this sense, Gómez-Rico et al. (2009) (GómezRico et al. 2009) also presented higher values of these parameters in EVOO following water stress treatment. Sena-Moreno et al. (2017) (Sena-Moreno et al. 2017) demonstrated greater oxidative stability in oil produced from a super-high-density olive grove (cv. Arbequina) when submitted to severe water stress. These researchers argued that this was due to an increase in antioxidant compounds and total polyphenols. Water stress in olive plants triggers a chemical reaction which reduces oxidative damage and stimulates the synthesis of phenols (Sánchez-Rodríguez et al. 2011). It is well known that these EVOO compounds are characterized by an antioxidant activity (Franco et al. 2014) that can increase oxidative stability. Cabrera-Bañegil et al. (2018) (CabreraBañegil et al. 2018) also observed an increase in total polyphenols in Arbequina variety olive trees with an irrigation deficit.

To sum up, taking into account all the previous results, there is a compromise between the olive oil production and its shelf life. Oliviculture sector has to assess how to proceed in order to take advantage of the current market.

\section{Olive Oil Excitation-Emission Matrices}

Due to the native fluorescence of olive oil, fluorescence spectroscopy was selected with discriminatory and quantitative purposes. A full EEM was recorded in order to obtain complete fluorescence information in relation to the olive oil sample. Figure 1A presents the complete EEM recorded for a single olive oil sample. As can be observed, the figure presents two fluorescence regions. The first region has an excitation maximum at $370 \mathrm{~nm}$ and two emission maxima at 470 and $500 \mathrm{~nm}$. The second region presented a very intensive maximum, occurring at $410 \mathrm{~nm}$ for excitation and $675 \mathrm{~nm}$ for emission. Other less intense maxima were found at higher excitation wavelengths. The obtained EEMs are similar to those found in existing literature (Guimet et al. 2004b; Jiménez-Carvelo et al. 2019; Lia et al. 2020). According to the conclusions of these prior studies, the first fluorescence band may be related to vitamins and oxidation products, whilst the second band appears to be correlated with chlorophyll derivatives.

In order to simplify outcomes, only the first band was examined in proceeding analysis. Figure 1B presents the EEMs pertaining to olive oil produced during the 2016/2017 crop season from Arbosana and Oliana variety fruit, respectively, according to different irrigation conditions. As can be observed, both EEMs from Arbosana and Oliana olive oils present an excitation maximum at $370 \mathrm{~nm}$ and two emission maxima at 470 and $500 \mathrm{~nm}$. Nevertheless, another maximum was found at approximately $330 \mathrm{~nm}$ of excitation and $440 \mathrm{~nm}$ of emission in Arbosana samples.

Further, the hydric regime had no effect on matrix physiognomy, although it was seen to affect the fluorescence intensity of signals obtained at 470 and $500 \mathrm{~nm}$. In both varieties, RDI samples presented higher fluorescence than control samples.

\section{Exploratory Analysis}

As can be seen in Fig. 1, olive oils obtained from different olives presented different EEMs. The utility of using these differences to distinguish between olive oils from different olive varieties and subjected to different irrigation treatments has been analysed using chemometric algorithms, such as PARAFAC and LDA. Firstly, an exploratory analysis was performed using PARAFAC. The 64 EEMs were arranged according to a three dimensional $64 \times 110 \times 22$ (samples $\times$ number of emission wavelengths $\times$ number of excitation wavelengths) structure. Then, this array was decomposed by PARAFAC, in order to select the optimal number of components. Three components were selected, accounting the $98.27 \%$ of the explained variance and having a core consistency criterion of 99 . 
Fig. 1 (A) Fluorescence contour plot of a full EEM for a single olive oil sample. (B) Fluorescence contour plot of olive oils produced during the 2016/2017 crop season from Arbosana and Oliana cultivars, according to irrigation treatments (control versus RDI)

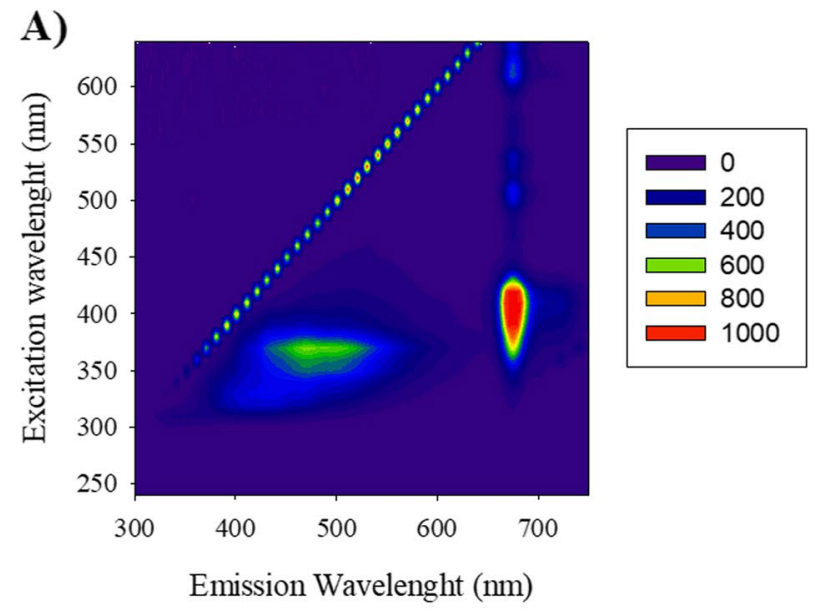

B)
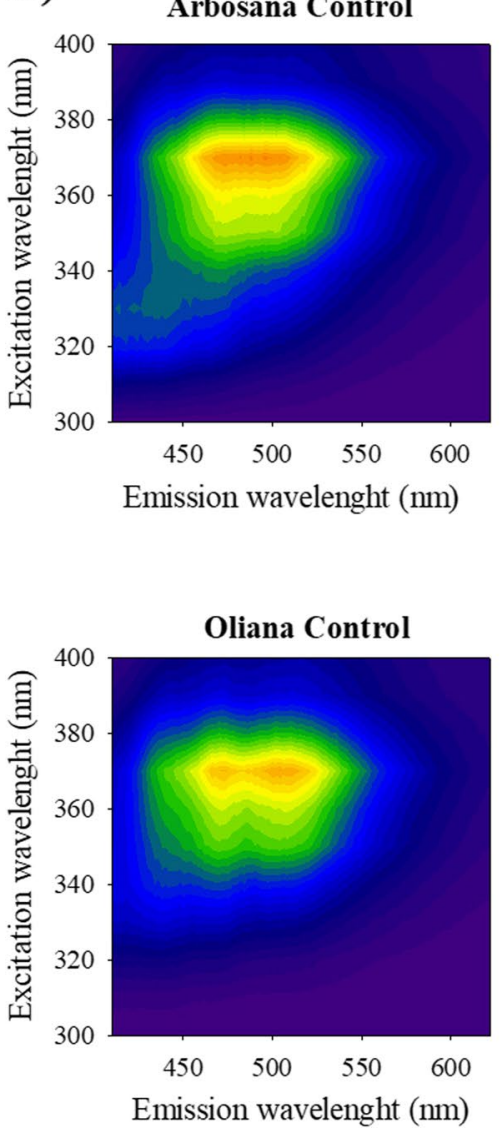
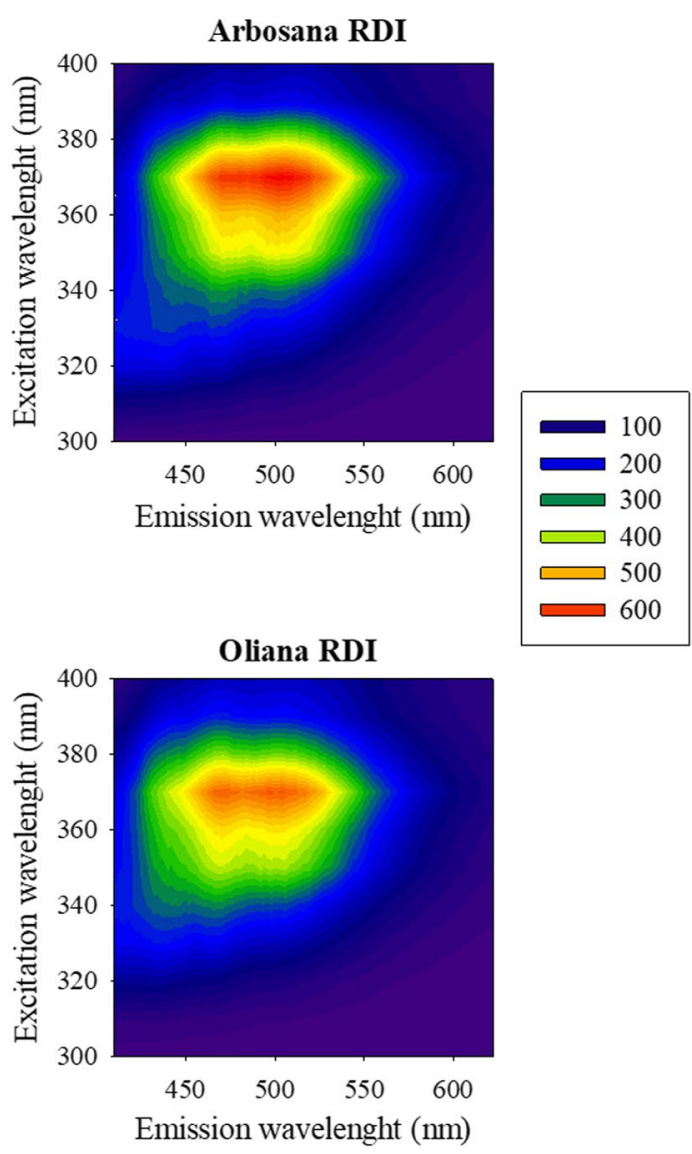

In PARAFAC, each component consists of two loading vectors (excitation and emission spectral data) and one score vector that provides information related to the constituent concentration. Excitation and emission PARAFAC loadings for the three components are shown in Fig. 2. These profiles corroborate the initial examination of fluorescence data and point to the types of fluorophores present in the samples, thus improving spectral interpretation.
The first component presents an excitation maximum at $370 \mathrm{~nm}$, a shoulder at $350 \mathrm{~nm}$ and two emission maxima at 475 and $500 \mathrm{~nm}$. The maximum intensity of the second component is also seen at an excitation wavelength of $370 \mathrm{~nm}$ and the shoulder at $350 \mathrm{~nm}$, whilst maximum emission wavelength is found at $520 \mathrm{~nm}$. It is difficult to characterize these bands given that many endogenous fluorescent molecules are seen in the $400-600 \mathrm{~nm}$ spectral range. According 
Fig. 2 (A) Excitation and emission PARAFAC loadings for the overall set of olive oil samples

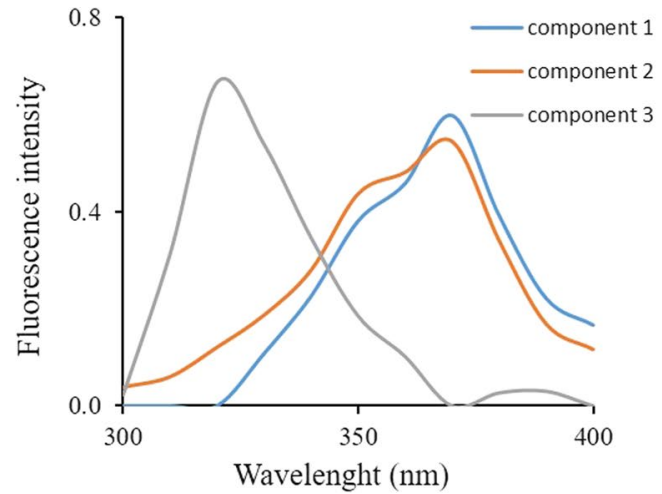

to Kyriakidis and Skarkalis, the first component could be attributed to the products of fatty acid oxidation (Kyriakidis and Skarkalis 2000). With respect to the second component, emission spectra with a maximum centred at $524 \mathrm{~nm}$ have previously been described in olive oil. This has been associated with the presence of vitamin B2 and with other compounds with similar chemical structures, such as flavinadenine dinucleotide (Zandomeneghi et al. 2005). This peak has also been detected in olive oil used in another study, where authors argued that it was, at least partly, explained by the presence of vitamin $\mathrm{E}$ derivatives (Kyriakidis and Skarkalis 2000).

The third component presents a maximum fluorescence intensity at $410 \mathrm{~nm}$, exciting at $320 \mathrm{~nm}$. In accordance with existing literature, this component is attributed to oxidation products (Guimet et al. 2004b).

\section{Discrimination According to Year of Cultivar, Variety and Irrigation Treatment}

\section{Discrimination Between Years}

As can be seen in Fig. 3, plotting the PARAFAC scores pertaining to the three components of all samples against each other enables olive oil samples from the 2016/2017 crop season to be clearly differentiated from 2017/2018 samples. In view of the good separation obtained with PARAFAC, a discrimination model was then built by applying a supervised algorithm. LDA was applied to the PARAFAC score matrix in order to separate the two sample groups. This is achieved by establishing a linear discriminant function that maximizes the ratio between the class and within-class variances. As explained in Sect. 2.7, samples were split into two sets. The training set used a $44 \times 3$ matrix ( 44 samples [ 22 for each variety] $\times$ scores for the three factors) and a $44 \times 1 \mathrm{Y}$ matrix composed of binary digits which identified the crop season to which each sample belonged. The validation set contained the remaining 20 samples (10 of each variety). Table 2 presents venetian blind cross-validation outcomes and the validation

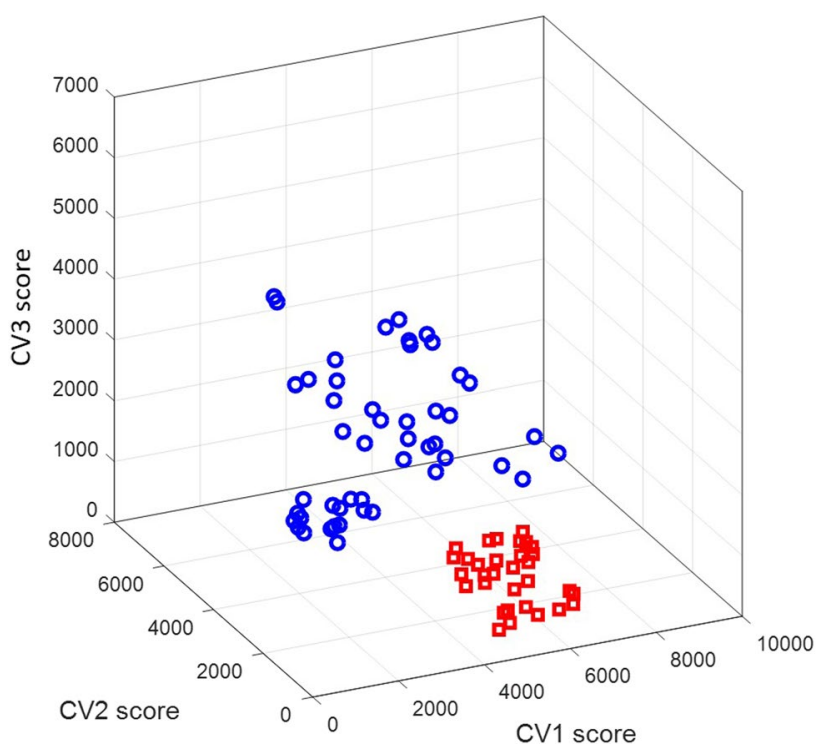

Fig. 3 PARAFAC scores for olives oil classification, with values pertaining to each crop season being presented separately. 2016/2017 crop season samples are plotted in blue, and 2017/2018 crop season samples are plotted in red

Table 2 Confusion matrix obtained through LDA-PARAFAC model for crop season discrimination. Values are expressed in number of samples

\begin{tabular}{llllll}
\hline & \multicolumn{3}{l}{ Predicted crop season } & \\
\cline { 2 - 3 } Real crop season & Cross-validation & & \multicolumn{2}{l}{ Validation } \\
\cline { 2 - 3 } \cline { 5 - 6 } \cline { 5 - 6 } $2016 / 2017$ & $2017 / 2018$ & & $2016 / 2017$ & $2017 / 2018$ \\
\hline $2016 / 2017$ & $\mathbf{2 2}$ & 0 & $\mathbf{1 0}$ & 0 \\
$2017 / 2018$ & 0 & $\mathbf{2 2}$ & 0 & $\mathbf{1 0}$ \\
\hline
\end{tabular}

Diagonal bold contains the number of correct assignments

confusion matrix for the PARAFAC-LDA model developed to discriminate between crop seasons. The classification model correctly classified all the cross-validation samples and test sets into their respective categories. Zero classification errors were recorded. 
Discrimination Between Varieties According to Crop Season

No visible trends were found among the olive oils of the two varieties when all the samples were employed together. Thus, data for each crop season were treated separately for the discrimination analysis of olive oils according to olive variety.

Firstly, in order to perform an exploratory analysis with PARAFAC, two different $32 \times 110 \times 22$ arrays (samples $\times$ number of emission wavelengths $\times$ number of excitation wavelengths) were built, with one pertaining to samples from 2016/2017 and the other pertaining to 2017/2018 olive oil samples. In both groups of samples, the CORCONDIA procedure suggested that three factors were sufficient to make up the model. It is noteworthy to state that, at this point, the loading profiles for both groups of samples were similar to those presented in Fig. 2 which used all samples.

With regard to PARAFAC scores. Figure 4 presents the three-dimensional plots for each of the sample groups. As can be seen, an adequate separation is observed in the two campaigns, being better in the 2017/18 campaign .

In view of the promising separation obtained using PARAFAC, LDA was applied employing the three PARAFAC factor scores as inputs. In this way, two classification models were built, with one pertaining to samples from the 2016/2017 crop season and the other to samples from 2017/2018. In both cases, the calibration set contained 22 samples (11 of each variety), and the validation set contained the remaining 10 samples ( 5 of each variety). Table 3 presents classification outcomes for both models. It presents a confusion matrix, with the numbers in the rows corresponding to actual classifications and columns corresponding to predicted classifications. Values along the matrix diagonal and presented in bold font correspond to the correct predictions made by LDA. The confusion matrix produced in relation to $2016 / 2017$ crop season samples has a $91 \%$ and a $100 \%$ of correct predictions in cross-validation and validation, respectively. In cross-validation, all Arbosana
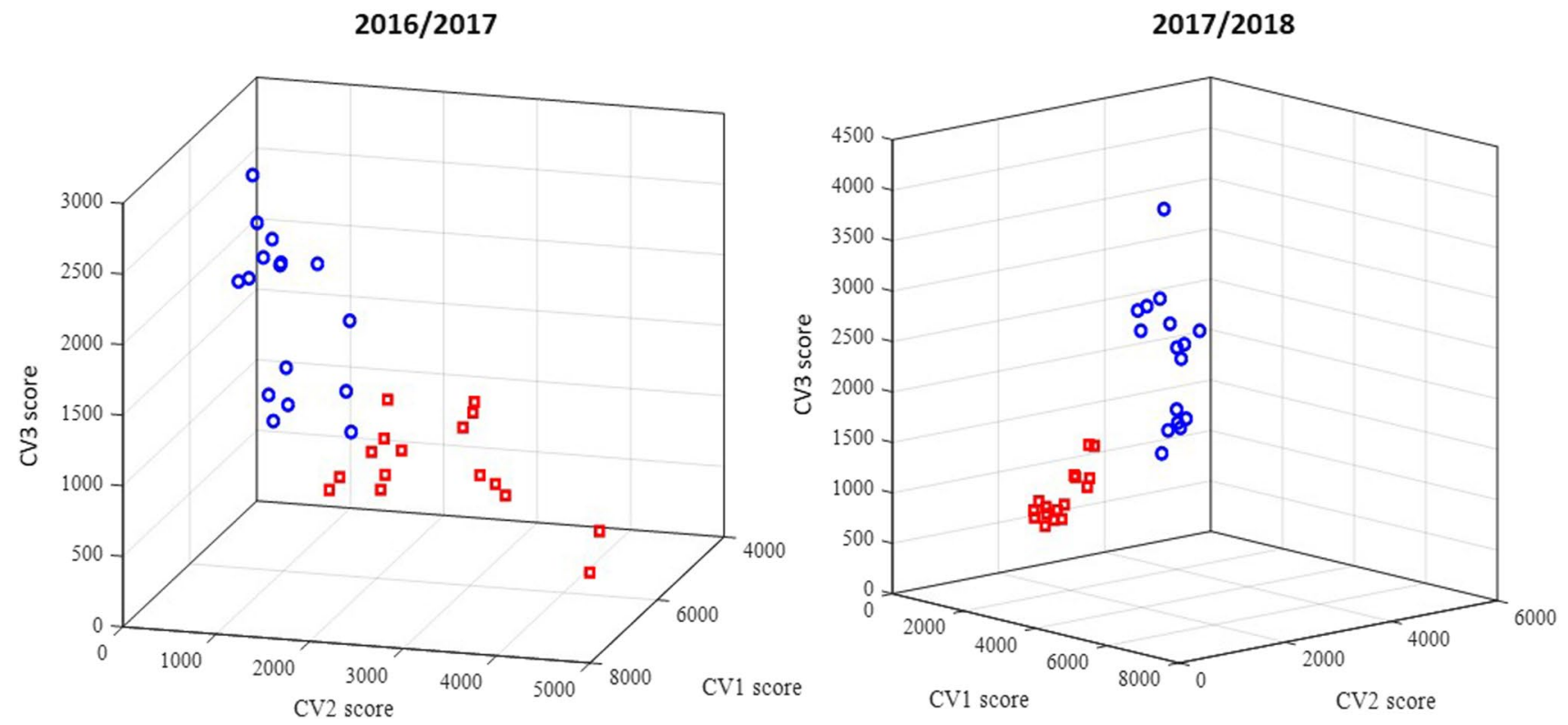

Fig. 4 PARAFAC scores for olive oils classification according to olive cultivar. Arbosana (red) and Oliana (blue)

Table 3 Confusion matrix obtained with LDAPARAFAC models for variety discrimination. Values are expressed in number of samples

\begin{tabular}{|c|c|c|c|c|c|c|c|c|}
\hline \multirow{4}{*}{ Real cultivar } & \multicolumn{8}{|c|}{ Predicted cultivar } \\
\hline & \multicolumn{4}{|l|}{$2016 / 2017$} & \multicolumn{4}{|c|}{$2017 / 2018$} \\
\hline & \multicolumn{2}{|c|}{ Cross validation } & \multicolumn{2}{|c|}{ Validation } & \multicolumn{2}{|c|}{ Cross-validation } & \multicolumn{2}{|c|}{ Validation } \\
\hline & Arbosana & Oliana & Arbosana & Oliana & Arbosana & Oliana & Arbosana & Oliana \\
\hline Arbosana & 11 & 0 & 5 & 0 & 11 & 0 & 5 & 0 \\
\hline Oliana & 2 & 9 & 0 & 5 & 0 & 11 & 0 & 5 \\
\hline
\end{tabular}

Diagonal bold contains the number of correct assignments 
variety samples were correctly classified; however, two Oliana variety samples were classified as belonging to the Arbosana variety. In contrast, all validation samples were correctly classified. With regards to the model constructed with samples from the 2017/2018 season, all samples were correctly assigned in both cross-validation and validation.

In consideration of these outcomes, it can be concluded that it is possible to correctly discriminate between olive oils produced from two different olive varieties by using fluorescence spectroscopy.

\section{Discrimination of Olive Oil from Irrigated and Non-irrigated Cultivars}

A similar analysis was conducted for the discrimination of olive oil subjected to different irrigation conditions (control and RDI). In this case, four different PARAFAC models were built, with one pertaining to each variety and crop season. A $16 \times 110 \times 22$ array was used for all models, and a total of three components was identified as optimum. Figure 5 shows the three-dimensional plots drawn from the three PARAFAC component scores. As can be seen,

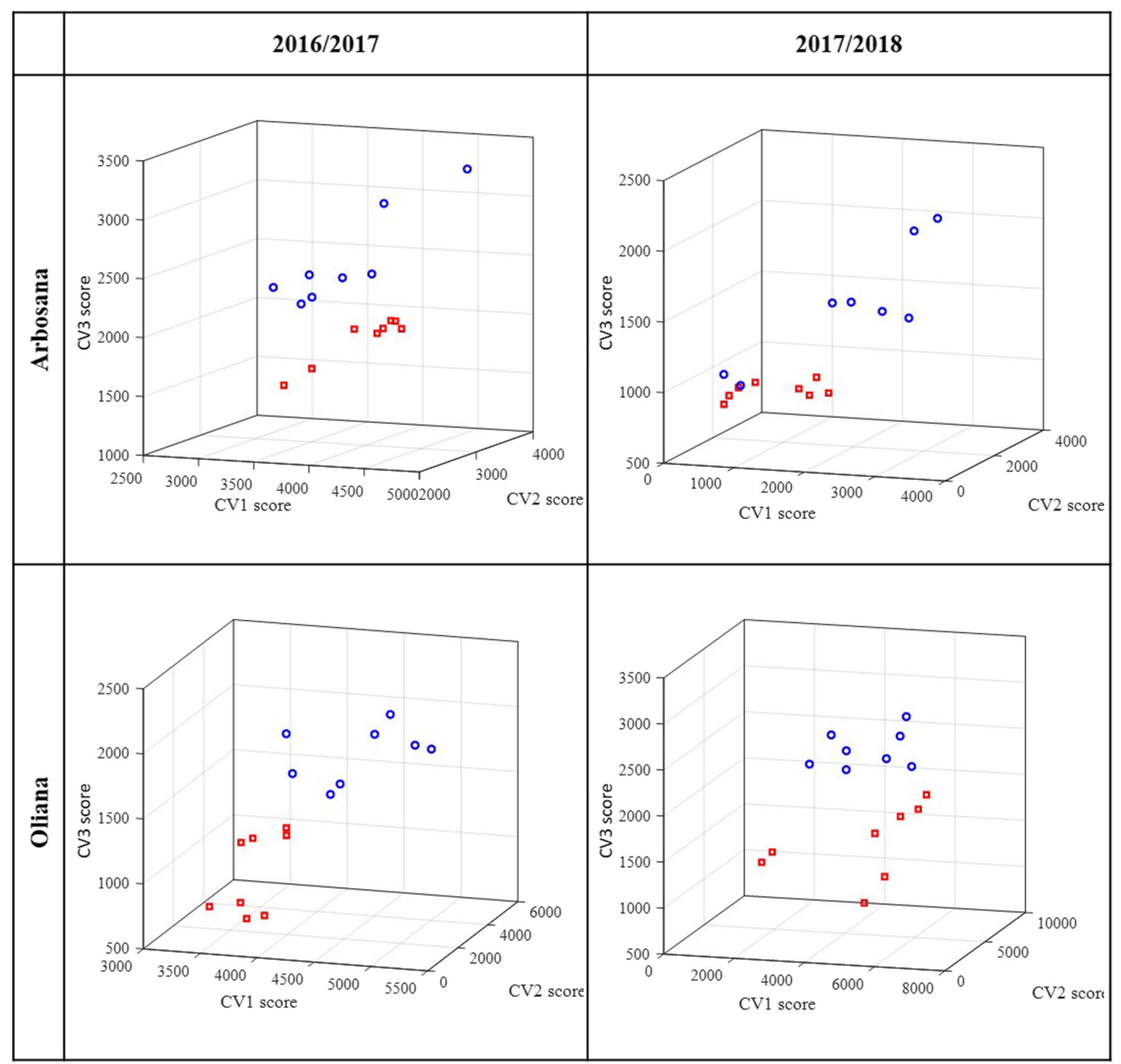

Fig. 5 PARAFAC scores for olive oil classification according to irrigation condition. RDI (red) and control (blue) 
Table 4 Confusion matrix obtained through PARAFACLDA model for irrigation condition discrimination. Values are expressed in number of samples

\begin{tabular}{|c|c|c|c|c|c|c|c|c|c|}
\hline \multicolumn{10}{|c|}{ Predicted irrigation condition } \\
\hline & & \multicolumn{4}{|c|}{$2016 / 2017$} & \multicolumn{4}{|c|}{$2017 / 2018$} \\
\hline \multirow{2}{*}{\multicolumn{2}{|c|}{$\begin{array}{l}\text { Real irrigation } \\
\text { condition }\end{array}$}} & \multicolumn{2}{|c|}{ Cross-validation } & \multicolumn{2}{|c|}{ Validation } & \multicolumn{2}{|c|}{ Cross-validation } & \multicolumn{2}{|c|}{ Validation } \\
\hline & & Control & $\overline{\text { RDI }}$ & Control & $\overline{\text { RDI }}$ & Control & $\overline{\text { RDI }}$ & Control & $\overline{\text { RDI }}$ \\
\hline \multirow[t]{2}{*}{ Arbosana } & Control & 6 & 0 & 2 & 0 & 4 & 1 & 3 & 0 \\
\hline & RDI & 0 & 5 & 0 & 3 & 2 & 4 & 1 & 1 \\
\hline \multirow[t]{2}{*}{ Oliana } & Control & 6 & 0 & 2 & 0 & 6 & 0 & 2 & 0 \\
\hline & RDI & 1 & 4 & 0 & 3 & 1 & 4 & 1 & 2 \\
\hline
\end{tabular}

Diagonal bold contains the number of correct assignments most samples are grouped according to their irrigation conditions.

Following this, in line with that previously conducted, a classification model was performed from the matrix produced from the three factor scores using LDA algorithm. In order to build each model, 11 of the 16 samples were selected for calibration, with the other 5 samples being selected for validation. The cross-validation and validation confusion matrices obtained for the four PARAFAC-LDA models are shown in Table 4. With regard to cross-validation, between 73 and $100 \%$ of the samples were correctly classified, whilst for validation, the percentage of correct predictions was between the 80 and $100 \%$. Almost all control samples were correctly assigned, and the majority of incorrectly classified samples belonged to RDI condition.

These results suggest that EEMs, combined with multivariate analysis, are a useful tool for discriminating between olive oils produced from olives submitted to different irrigation conditions.

Other authors have also previously shown that EEMs enabled the differentiation according to irrigation treatment,
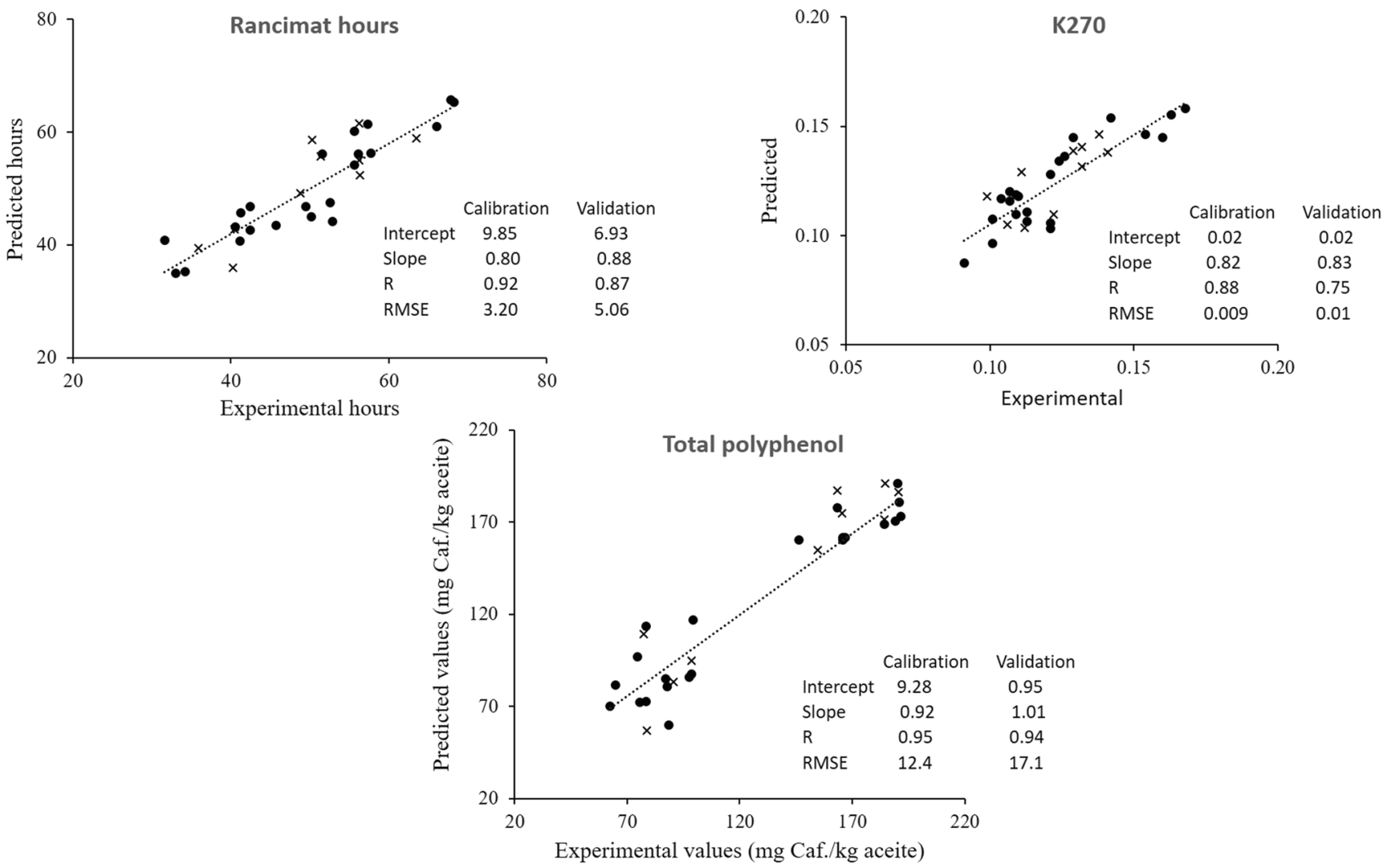

Fig. 6 Predicted versus experimental values for Rancimat hours, $\mathrm{K}_{270}$ and total polyphenol content in calibration (•) and validation samples (x). RMSE root-mean square error 
although these studies used olive paste and examined different fluorescence regions (Cabrera-Bañegil et al. 2018).

\section{Relationship Between Fluorescence and the Quality Index}

Considering the fact that the observed fluorescence may be related with oxidation products, the potential use of fluorescence spectroscopy to quantify important quality parameters of olive oil samples was examined. In this sense, although this fluorescence region is not characterized by polyphenols, total polyphenol content and two stability parameters were quantified.

U-PLS regression was chosen to establish quantitative models between the chemical values of parameters obtained through reference procedures (as indicated in Sect. 2.2) and EEMs. A U-PLS model was built using the calibration samples (22 samples) for each oxidative stability parameter and for total polyphenol content. First, the Haaland and Thomas criterion (Haaland and Thomas 1988) was employed to select the optimum number of components, as explained in Sect. 2.7. The optimum number of latent variables was six for all models. Following this, predictions were performed in relation to the validation samples ( 10 samples). Figure 6 presents predicted versus experimental values obtained according to the aforementioned methods, for Rancimat hours, $\mathrm{K}_{270}$ and total polyphenol content. A good correlation can be appreciated between the predicted values obtained from the fluorescence data and the experimental values. Figure 6 also shows the statistical parameters for both cross-validation and validation samples. Correlation coefficients in the crossvalidation for Rancimat hours, K270 and total polyphenol content were $0.92,0.88$ and 0.95 , respectively. These values were $0.87,0.75$ and 0.94 , respectively, in the validation.

From these results, it can be concluded that EEM analysis, together with U-PLS, offers a powerful tool for the determination of Rancimat hours, K270 and total polyphenol content in olive oil.

\section{Conclusions}

The present study demonstrated that fluorescence spectroscopy combined with multiway algorithms offers a powerful tool for olive oil discrimination. Three-way data were first analysed by using PARAFAC. Following this, scores for the first three components were used in LDA, enabling discrimination between samples according to the crop season (2016/2017 versus 2017/2018) and according to the variety within each crop season.
Moreover, EEMs combined with PARAFAC-LDA enabled the discrimination of olive oil samples subjected to control irrigation and regulated deficit irrigation conditions. In a practical sense, this could be used to indicate irrigation problems during the growing season. Furthermore, this nondestructive technique could be used by the olive oil industry to obtain information of samples with different oxidative stability, allowing the commercialization of the product at the optimum moment with the objective to ensure the quality of the olive oils.

In addition, the combination of fluorescence spectroscopy with second-order U-PLS algorithm allowed important quality parameters, including Rancimat hours, $\mathrm{K}_{270}$ and total polyphenol content, to be quantified in olive oil without sample pre-treatment or chemical reagent use.

In consideration to these outcomes, it can be concluded that fluorescence spectroscopy is a fast, powerful and nondestructive tool for olive oil discrimination and the quantification of important quality parameters, without the use of reagents.

Funding Open Access funding provided thanks to the CRUE-CSIC agreement with Springer Nature. Antonio Fernández thanks Junta de Extremadura and European Social Fund for the Garantia Juvenil contract (reference: TE-0078-19) at the CICYTEX-INTAEX research centre. Isabel Duran thanks the financial support received from the Ministerio de Ciencia e Innovación of Spain (Project PID2020-112996GB-100) and by Junta de Extremadura (Project IB20016 and Research Group FQM003), both co-financed by the Fondo Europeo de Desarrollo Regional.

\section{Declarations}

Ethics Approval This article does not contain any studies with human participants or animals performed by any of the authors.

Informed Consent Informed consent not applicable.

Conflict of Interest The authors declare no competing interests.

Open Access This article is licensed under a Creative Commons Attribution 4.0 International License, which permits use, sharing, adaptation, distribution and reproduction in any medium or format, as long as you give appropriate credit to the original author(s) and the source, provide a link to the Creative Commons licence, and indicate if changes were made. The images or other third party material in this article are included in the article's Creative Commons licence, unless indicated otherwise in a credit line to the material. If material is not included in the article's Creative Commons licence and your intended use is not permitted by statutory regulation or exceeds the permitted use, you will need to obtain permission directly from the copyright holder. To view a copy of this licence, visit http://creativecommons. org/licenses/by/4.0/. 


\section{References}

Ballabio D, Consonni V (2013) Classification tools in chemistry. Part 1: Linear models PLS-DA. Anal Methods 5:3790-3798. https:// doi.org/10.1039/c3ay40582f

Bassani B, Rossi T, De Stefano D et al (2016) Potential chemopreventive activities of a polyphenol rich purified extract from olive mill wastewater on colon cancer cells. J Funct Foods 27:236-248. https://doi.org/10.1016/j.jff.2016.09.009

Berrueta LA, Alonso-Salces RM, Héberger K (2007) Supervised pattern recognition in food analysis. J Chromatogr A 1158:196-214. https://doi.org/10.1016/j.chroma.2007.05.024

Bro R (1997) PARAFAC. Tutorial and applications. Chemom Intell Lab Syst 38:149-171. https://doi.org/10.1016/S0169-7439(97) 00032-4

Bro R, Kiers HAL (2003) A new efficient method for determining the number of components in PARAFAC models. J Chemom 17:274-286. https://doi.org/10.1002/cem.801

Cabrera-Bañegil M, Martín-Vertedor D, Boselli E, Durán-Merás I (2018) Control of olive cultivar irrigation by front-face fluorescence excitation-emission matrices in combination with PARAFAC. J Food Compos Anal 69:189-196. https://doi.org/ 10.1016/j.jfca.2018.01.021

Dagdelen A, Tümen G, Özcan MM, Dündar E (2013) Phenolics profiles of olive fruits (Olea europaea L.) and oils from Ayvalik, Domat and Gemlik varieties at different ripening stages. Food Chem 136:41-45. https://doi.org/10.1016/j.foodchem.2012.07. 046

Delgado-Adámez J, Nieves Franco Baltasar M, Ayuso Yuste MC, Martín-Vertedor D (2014) Oxidative stability, phenolic compounds and antioxidant potential of a virgin olive oil enriched with natural bioactive compounds. J Oleo Sci 63:55-65. https:// doi.org/10.5650/jos.ess 13114

Difonzo G, Russo A, Trani A et al (2017) Green extracts from Coratina olive cultivar leaves: antioxidant characterization and biological activity. J Funct Foods 31:63-70. https://doi.org/10. 1016/j.jff.2017.01.039

Domínguez Manzano J, Muñoz de la Peña A, Durán Merás I (2019) Front-face fluorescence combined with second-order multiway classification, based on polyphenol and chlorophyll compounds, for virgin olive oil monitoring under different photo- and thermal-oxidation procedures. Food Anal Methods 12:1399-1411. https://doi.org/10.1007/s12161-019-01471-1

Durán Merás I, Domínguez Manzano J, Airado Rodríguez D, Muñoz de la Peña A (2018) Detection and quantification of extra virgin olive oil adulteration by means of autofluorescence excitationemission profiles combined with multi-way classification. Talanta 178:751-762. https://doi.org/10.1016/j.talanta.2017.09.095

EEC (2003) Commision regulation (EC) No 1989/2003 amending Regulation (EEC) No 2568/91 on the characteristics of olive oil and olive-pomace oil and on the relevant methods of analysis. Off J Eur Commun L295:57-77

Franco MN, Galeano-Díaz T, López Ó et al (2014) Phenolic compounds and antioxidant capacity of virgin olive oil. Food Chem 163:289-298. https://doi.org/10.1016/j.foodchem.2014.04.091

Gómez-Rico A, Salvador MD, Fregapane G (2009) Virgin olive oil and olive fruit minor constituents as affected by irrigation management based on SWP and TDF as compared to ETc in medium-density young olive orchards (Olea europaea L. cv. Cornicabra and Morisca). Food Res Int 42:1067-1076. https:// doi.org/10.1016/j.foodres.2009.05.003

Guimet F, Boqué R, Ferré J (2005a) Study of oils from the protected denomination of origin "Siurana" using excitation-emission fluorescence spectroscopy and three-way methods of analysis.
Grasas Aceites 56:292-297. https://doi.org/10.3989/gya.2005. v56.i4.95

Guimet F, Boqué R, Ferré J (2004a) Cluster analysis applied to the exploratory analysis of commercial Spanish olive oils by means of excitation-emission fluorescence spectroscopy. J Agric Food Chem 52:6673-6679. https://doi.org/10.1021/jf040169m

Guimet F, Ferré J, Boqué R et al (2005b) Excitation-emission fluorescence spectroscopy combined with three-way methods of analysis as a complementary technique for olive oil characterization. J Agric Food Chem 53:9319-9328. https://doi.org/10. $1021 / \mathrm{jf051237n}$

Guimet F, Ferré J, Boqué R (2005c) Rapid detection of olive-pomace oil adulteration in extra virgin olive oils from the protected denomination of origin "Siurana" using excitation-emission fluorescence spectroscopy and three-way methods of analysis. Anal Chim Acta 544:143-152. https://doi.org/10.1016/j.aca. 2005.02.013

Guimet F, Ferré J, Boqué R, Rius FX (2004b) Application of unfold principal component analysis and parallel factor analysis to the exploratory analysis of olive oils by means of excitationemission matrix fluorescence spectroscopy. Anal Chim Acta 515:75-85. https://doi.org/10.1016/j.aca.2004.01.008

Gutiérrrez F (1989) Determinación de la estabilidad oxidativa de aceites de oliva vírgenes: comparación entre el método AOM y el método Rancimat. Grasas Aceites 40:1-5

Haaland DM, Thomas EV (1988) Partial least-squares methods for spectral analyses. 1. Relation to other quantitative calibration methods and the extraction of qualitative information. Anal Chem 1193-1202. https://doi.org/10.1021/ac00162a020

Jiménez-Carvelo AM, Lozano VA, Olivieri AC (2019) Comparative chemometric analysis of fluorescence and near infrared spectroscopies for authenticity confirmation and geographical origin of Argentinean extra virgin olive oils. Food Control 96:22-28. https://doi.org/10.1016/j.foodcont.2018.08.024

Kyriakidis NB, Skarkalis P (2000) Fluorescence spectra measurement of olive oil and other vegetable oils. J AOAC Int 83:1435-1439. https://doi.org/10.1093/jaoac/83.6.1435

Lavee S, Hanoch E, Wodner M, Abramowitch H (2007) The effect of predetermined deficit irrigation on the performance of $\mathrm{cv}$. Muhasan olives (Olea europaea L.) in the eastern coastal plain of Israel. Sci Hortic (amsterdam) 112:156-163. https://doi.org/10. 1016/j.scienta.2006.12.017

Lia F, Formosa JP, Zammit-Mangion M, Farrugia C (2020) The first identification of the uniqueness and authentication of Maltese extra virgin olive oil using 3D-fluorescence spectroscopy coupled with multi-way data analysis. Foods 9:498. https://doi.org/ 10.3390/foods 9040498

Machado M, Felizardo C, Fernandes-Silva AA et al (2013) Polyphenolic compounds, antioxidant activity and 1-phenylalanine ammonia-lyase activity during ripening of olive cv. "Cobrançosa" under different irrigation regimes. Food Res Int 51:412-421. https://doi. org/10.1016/j.foodres.2012.12.056

Martínez JM, Muñoz E, Alba J, Lanzón A (1975) Informe sobre utilización del Analizador de Rendimientos "Abencor. Grasas Aceites 26:379-385

Olivieri AC, Wu HL, Yu RQ (2009) MVC2: A MATLAB graphical interface toolbox for second-order multivariate calibration. Chemom Intell Lab Syst 96:246-251. https://doi.org/10.1016/j. chemolab.2009.02.005

Orgaz F, Testi L, Villalobos FJ, Fereres E (2006) Water requirements of olive orchards-II: Determination of crop coefficients for irrigation scheduling. Irrig Sci 24:77-84. https://doi.org/10.1007/ s00271-005-0012-x

Prieto MH, Pérez-Rodríguez JM, Lara Carrasco E (2016) Olivicultura de regadío en Extremadura: del olivar tradicional al 
superintensivo. In: CB F (ed) La agricultura y la ganadería extremeñas, pp 91-106

Sánchez-Rodríguez E, Moreno DA, Ferreres F et al (2011) Differential responses of five cherry tomato varieties to water stress: changes on phenolic metabolites and related enzymes. Phytochemistry 72:723-729. https://doi.org/10.1016/j.phytochem.2011.02.011

Sena-Moreno E, Pérez-Rodríguez JM, De Miguel C et al (2017) Pigment profile, color and antioxidant capacity of arbequina virgin olive oils from different irrigation treatments. J Am Oil Chem Soc 94:935-945. https://doi.org/10.1007/s11746-017-3008-9

Sikorska E, Khmelinskii IV, Sikorski M et al (2008) Fluorescence spectroscopy in monitoring of extra virgin olive oil during storage. Int J Food Sci Technol 43:52-61. https://doi.org/10.1111/j. 1365-2621.2006.01384.x

Squeo G, Caponio F, Paradiso VM et al (2019) Evaluation of total phenolic content in virgin olive oil using fluorescence excitationemission spectroscopy coupled with chemometrics. J Sci Food Agric 99:2513-2520. https://doi.org/10.1002/jsfa.9461

Tena N, Aparicio R, García-González DL (2012) Chemical changes of thermoxidized virgin olive oil determined by excitation-emission fluorescence spectroscopy (EEFS). Food Res Int 45:103-108. https://doi.org/10.1016/j.foodres.2011.10.015
Terzuoli E, Giachetti A, Ziche M, Donnini S (2016) Hydroxytyrosol, a product from olive oil, reduces colon cancer growth by enhancing epidermal growth factor receptor degradation. Mol Nutr Food Res 60:519-529. https://doi.org/10.1002/mnfr.201500498

Uceda M, Frías L (1975) Harvest dates. Evolution of the fruit oil content, oil composition and oil quality. In: Proceedings of the II Seminario Oleícola Internacional. Córdoba, Spain, pp 125-128

Wold S, Geladi P, Esbensen K, Öhman J (1987) Multi-way principal components and pls-analysis. J Chemom 1:41-56. https://doi.org/ 10.1002/cem.1180010107

Zandomeneghi M, Carbonaro L, Caffarata C (2005) Fluorescence of vegetable oils: olive oils. J Agric Food Chem 53:759-766. https:// doi.org/10.1021/jf048742p

Publisher's Note Springer Nature remains neutral with regard to jurisdictional claims in published maps and institutional affiliations. 